\section{EFICACIA DE LOS MAPAS \\ CONCEPTUALES COMO \\ RECURSO DIDÁCTICO EN EL \\ APRENDIZAJE DE LAS CIENCIAS \\ SOCIALES EN LA ESCUELA \\ ACADÉMICO PROFESIONAL DE \\ TURISMO, HOTELERÍA Y \\ GASTRONOMÍA DE LA UNIVERSIDAD \\ ALAS PERUANAS}

EFFECTIVENESS OF MIND MAPS AS

TEACHING RESOURCE IN THE

LEARNING PROCESS OF SOCIAL

SCIENCES IN THE PROFESSIONAL

ACADEMIC SCHOOL OF TOURISM,

HOSPITALITY AND CUISINE OF

PERUVIAN UNIVERSITY ALAS

PERUANAS

Mg. Rafael Antonio Garay Argandoña*

\section{RESUMEN}

Objetivo. El objetivo de la Investigación fue Determinar que el desconocimiento del manejo de los mapas conceptuales como recurso didáctico influye en el aprendizaje de las Ciencias Sociales en los estudiantes del II ciclo de la Escuela Académico Profesional de Turismo Hotelería y Gastronomía UAP Materiales y Métodos. Para la selección de la muestra se consideró a los estudiantes del II ciclo de la escuela, la técnica de muestreo fue sistemático, se trabajó desarrollando clases haciendo uso de mapas conceptuales en la asignatura de historia de la cultura se aplicó la prueba estadística de hi2 teniendo en cuenta la relación de variables Resultados. Como el valor calculado es mayor al de la tabla (24.894 > 16.919), la hipótesis nula es rechazada, aceptándose la hipótesis alterna $\mathrm{H} 1$, con lo cual queda demostrada la hipótesis. Conclusión. El manejo de los mapas conceptuales como recurso didáctico influye significativamente en el aprendizaje de los alumnos, produce un auténtico aprendizaje, porque proporciona conceptos de manera coherente y no arbitraria, "construyendo", de manera sólida los conceptos

\section{ABSTRACT}

Objective. The aim of the research was to determine that the lack of management of mind maps as a teaching resource influences in the learning of Social Sciences students in cycle II of the Professional Academic School of Tourism Hospitality and Gastronomy in Alas Peruanas University. For the selection of the sample, the students of the second cycle school were considered, the sampling technique was systematic, classes were developed making use of concept maps in the history of culture subject statistic hi2 tests were applied taking in account the variables relation. Results

As the calculated value is bigger that the table $(24,894>16,919)$, the null hypothesis is rejected accepting the alternative hypothesis $\mathrm{H} 1$ whereupon, the hypothesis ends up being demonstrated.

In conclusion. The management of mind maps as a teaching resource influences significantly on the student learning process, It produces an effective learning because it provides concepts in a coherent and not arbitrary way by "building" concepts solidly.

\section{INTRODUCCIÓN}

- En el Proceso de Enseñanza Aprendizaje los medios de enseñanza constituyen un factor clave dentro del proceso Didáctico. Ellos favorecen que la comunicación bidireccional que existe entre los protagonistas pueda establecerse de manera más afectiva, en tal sentido la presente investigación titulada "Eficacia de los Mapas Conceptuales como Recurso Didáctico en el Aprendizaje de las Ciencias Sociales. Fue elaborada para apoyar la efectividad del trabajo del profesor y elevar la motivación de los estudiantes en el proceso de enseñanza aprendizaje. Es importante conocer el manejo de los mapas conceptuales que constituyen un instrumento pedagógico poderoso y que permiten al alumno una 
mejor comprensión del objeto de estudio. En este contexto, el empleo de los mapas conceptuales permite al profesor de Ciencias Sociales tener una clara visión de las principales funciones que pueden desarrollar en el proceso de enseñanza- aprendizaje; estas funciones son:

- Función Motivadora, en donde se trata de acercar el Aprendizaje a los intereses de los Alumnos y de contextualizarlos social y culturalmente, superando así el verbalismo como única vía.

- Función Configuradora, de la relación Cognitiva según el medio, el tipo de operación mental. Función Facilitadora de acción deductiva; por que los Mapas Conceptuales facilita la organización de las experiencias de los Aprendizajes, actuando como guías no solo en cuanto nos colocan en contacto con los contenidos, sino también en cuanto que requieren la realización de un trabajo con el propio medio.

- Función Formativa, los Mapas conceptuales permiten y provocan la aparición y expresiones de emociones, informaciones y valores que transmiten diversas modalidades de relación, cooperación o comunicación.

El Objetivo fundamental de la Investigación es Valorar los Mapas Conceptuales para fomentar en los estudiantes destrezas y habilidades intelectuales que le permitan la comprensión profunda de textos. Así mismo se busca que la aplicación de los mapas conceptuales desarrolle en los alumnos su pensamiento crítico.

\section{MATERIALES Y MÉTODOS}

La presente investigación es de tipo Sustantivo, Descriptivo co-relacional de la problemática generada por la eficiencia de los mapas conceptuales como recursos didácticos en el aprendizaje de las ciencias sociales.

De acuerdo a los objetivos y a las variables establecidas, el estudio que se realizará reúne las características que permiten ubicarlo en la modalidad de investigación de campo de carácter descriptivo co-relacional. Parte de una observación que evidenciará las características del universo de investigación, y señalará formas de conducta y actitudes del mismo. Al realizar la observación directa, los datos que se presentarán son de tipo primario, entonces, se puede decir que el estudio se ubica dentro de la investigación de campo descriptiva co-relacional.

La muestra estuvo conformada por 64 estudiantes del II ciclo de la EAP Turismo, Hotelería y Gastronomía, en la cual se aplicó una encuesta conformada por quince ítems, destacando el desconocimiento de la estrategia como le relación de los Mapas Conceptuales con las capacidades fundamentales de las Ciencias Sociales.

\section{RESULTADOS}

Considerando las respuestas de los alumnos se obtuvo:

Gráfico 01: Mapas conceptuales - nivel de rendimiento

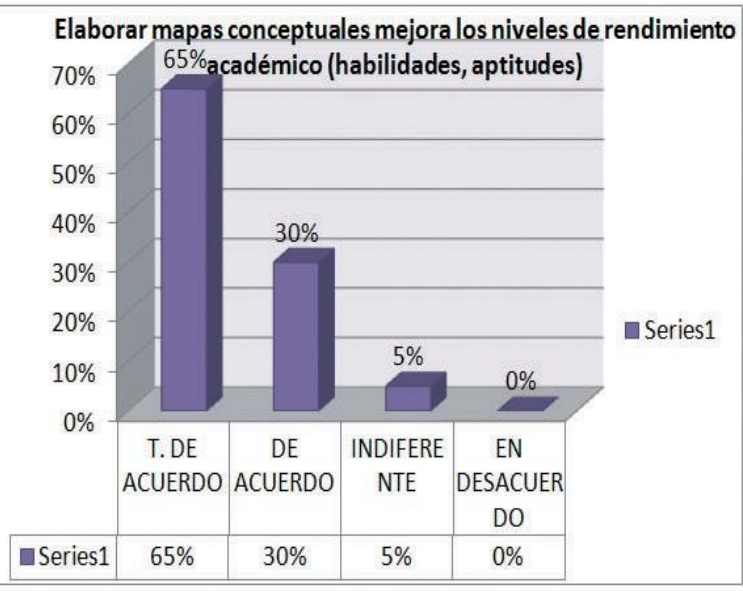

Fuente: Elaboración propia

\section{Interpretación}


Se demuestra un alto índice de reconocimiento por parte de los docentes en un $65 \%$ y un $30 \%$ estar de acuerdo que la influencia en la construcción de mapas conceptuales, considerados como instrumentos de evaluación, enseñanza y aprendizaje, tienen en el desarrollo aprendizaje significativo frente al aprendizaje memorístico-mecánico, y también valorar el mejoramiento de los niveles de rendimiento académico El análisis de la evolución de los mapas conceptuales construidos por los alumnos en tres fases: inicial individual, final individual y compartidos en grupos, muestra que los alumnos han aprendido significativamente y paralelamente a este hecho, los resultados obtenidos en la aplicación demuestran que ha tenido lugar un incremento en la inteligencia, puesto en evidencia por la evolución del cociente intelectual $(\mathrm{Cl})$ y de la llamada inteligencia fluida. Sólo un 5\% desconoce su valiosa importancia.

Cuadro 01: Tabla de frecuencias observadas de la encuesta a los alumnos

\begin{tabular}{|c|c|c|c|c|c|c|}
\hline & \multicolumn{5}{|c|}{ Mash Conceduds } \\
\hline & & TDA & $D A$ & $\overline{E D}$ & ETD & TOTAL \\
\hline \multirow{5}{*}{$\begin{array}{l}\text { Ancendizie } \\
\text { Sonifodivo }\end{array}$} & TDA & 15 & 3 & 1 & 2 & 21 \\
\hline & $\overline{D A}$ & 3 & 16 & 2 & 0 & 21 \\
\hline & ED & 3 & 4 & 2 & 4 & 13 \\
\hline & ETD & 1 & 4 & 3 & 1 & 9 \\
\hline & TOTAL & 22 & 27 & 8 & 7 & 64 \\
\hline
\end{tabular}

Fuente: Elaboración propia

Cuadro 02: Tabla de frecuencias esperadas de la encuesta a los alumnos

\begin{tabular}{|c|c|c|c|c|c|c|}
\hline & \multicolumn{5}{|c|}{ 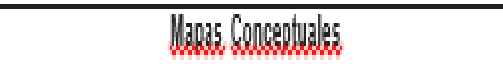 } \\
\hline & & TDA & DA & ED & ETD & TOTAL \\
\hline \multirow{5}{*}{ 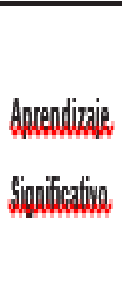 } & TDA & 7.2180 & 8,8594 & 26260 & 2.2969 & 21 \\
\hline & DA & 7.2180 & 8.8594 & 26250 & 22869 & 21 \\
\hline & ED & 4.486 & 5.444 & 1.6260 & 1.1219 & 13 \\
\hline & ETD & 3.093 & 37969 & 1.1260 & 0.9844 & 9 \\
\hline & TOTAL & 22 & 27 & 8 & 7 & 64 \\
\hline
\end{tabular}

\section{Fuente: Elaboración propia}

De los resultados de la encuesta a los alumnos, considerando las preguntas referentes a las variables Mapas Conceptuales y Aprendizaje de las Ciencias Sociales

De las tablas de distribución de jicuadrada para un nivel de confianza de 0.05 , se obtiene el valor de $\mathrm{Xx}=16.919$. Se acepta la hipótesis nula Ho si el valor calculado es menor o igual a 16.919. Como el valor calculado es mayor al de la tabla (34.1724 > 16.919), la hipótesis nula es rechazada, aceptándose la hipótesis alterna $\mathrm{H} 1$, con lo cual queda comprobada la hipótesis.

\section{DISCUSIÓN}

Se observa que el manejo de los mapas conceptuales como recurso didáctico influye significativamente en el aprendizaje de los alumnos, produce un auténtico aprendizaje, porque proporciona conceptos de manera coherente y no arbitraria, "construyendo", de manera sólida los conceptos, interconectando unos con los otros en forma de red de conocimiento. De modo que el alumnado aprende, identifica el campo próximo del cual puede aprender, decide los grados de dificultad que presenta y las conexiones pertinentes para conseguir la coherencia del aprendizaje. Los mapas conceptuales son un instrumento potente para conseguirlo.

Así mismo existe un desconocimiento de un $20 \%$ del uso y el manejo de los mapas conceptuales esto les permite memorizar mecánicamente los conceptos sin relacionarlos con las ideas que los alumnos tienen el área de ciencias sociales

Al igual que la investigación hecha por Revilla en la universidad de VeracruzMéxico coincidimos que los Mapas Conceptuales es una estrategia que 
favorece en una metodología activa donde el estudiante es protagonista de su propio aprendizaje.

\section{REFERENCIAS BIBLIOGRAFICAS.}

1. Alvares $y$ otros (2002). Enseñar para aprender: Procesos Estratégicos. Madrid España: CCS.

2. Alonso, J. (1991) Motivación y aprendizaje en el aula: Cómo enseñar a pensar. Madrid: Santillana.

3. Anderson, J. R. (1983) La arquitectura del conocimiento. Cambridge: Harvard University Press.

4. Araujo, J. B. y Chadwick, C. B. (1988) Tecnología educacional: Teorías de instrucción. Barcelona: Paidós Ibérica, S. A.

5. Ausubel, D. P., Novak, J. D., Hanesian, H. (1983) Psicología educativa.

México: Trillas.

6. Andina, M. y Gerardo A. S. (1992) Aprendizaje de las Ciencias Sociales. Argentina

:El Ateneo.

7. Barnes, B. R. y Clawson, E. U. (1975) El avance de las organizaciones. Review of Educational Research, 45 637-659.

8. Bruner, T. (1998) El Libro de los Mapas Mentales. España: Urano.

9. Calero, M. (1999) Aprender a Aprender con Mapas Conceptuales. Lima:San Marcos.

10. Castillo, S. (2005) Enseñar a Estudiar:Aprende a aprender. España:Pearson Educación S.A.

11. Castillo D,J.(s.f.) Formación
Ciudadana: Aprendizaje de Nueva Historia, Guía Metodológica. Lima: (s.n.)

12. Flores, $M . \quad H . \quad$ (1999) Inteligencias Múltiples en el Aula. Lima: San Marcos.

13. Flores, M. H. (1999) Mapas Conceptuales en el aula. Lima: San Marcos.

14. González, F. M. y Novak, J. D. (1993) Aprendizaje significativo: Técnicas y aplicaciones. Madrid:Cincel.

15. Guillén, L. A. (2007) Introducción a las Ciencias Sociales: curso del I. Ciclo 2007-I Lima: CEMED Universidad Nacional de Educación La Cantuta

16. Hernández, P. y García, L. A. (1983) Objetivos educacionales $y$ perfiles cognitivos en la evaluación académica. Memoria de licenciatura. Tenerife: Dpto. de Psicología Educativa, Evolutiva y Psicobiología. Universidad de la Laguna.

17. Novak, J. y Gowin, D.B. (1998) Aprendiendo a Aprender. España:Martínez Roca S.A.

18. Novak, J. (1991) Ayudar a los alumnos a aprender: cómo aprender la enseñanza de las Ciencias. 9 (3), 215-228

19. Novak, J. (1992) Teoría y práctica de la educación. Madrid: Alianza Universidad.

20. Pinillos, J. L. (1977) Principios de psicología. Madrid: Alianza Editorial.

21. Pozo, J. I. (1989) Introducción a la psicología cognitiva. Madrid: Alianza Psicología.

22. Pozo, J. L. (1996) Aprendices y 
maestros. Madrid: Alianza Editorial.

23. Soto, B. A. (2000) Los Organizadores del Conocimiento. Huancayo:Bachillerato UNCP.

24. Soto, B.A. (2000) Los Procesos Cognitivos en el Aprendizaje. Huancayo: Razuwillca ICIICEE, Maestro Innovador.

25. Vega de, M. (1984) Introducción a la psicología cognitiva. Madrid:Alianza Psicología. Valdivia, M. y Robles M. (1997) Decisiones laborales en las economías rurales del Perú. Revista GRADE. Notas para el Debate. 14 () 